\section{The prognostic value of electroencephalography in epilepsy: a long-term follow-up study}

\author{
Stylianos Gatzonis, ${ }^{1}$ \\ Nikolaos Triantafyllou, ${ }^{2}$ Maria Kateri, ${ }^{3}$ \\ Anna Siatouni, ${ }^{1}$ Elias Angelopoulos, ${ }^{4}$ \\ Vasilios K. Kimiskidis ${ }^{5}$ \\ 'Department of Neurosurgery, Athens \\ Medical School, Evangelismos Hospital; \\ 2Department of Neurology, Athens \\ Medical School, Eginition Hospital; \\ ${ }^{3}$ Department of Statistics and Insurance \\ Science, University of Piraeus; \\ ${ }^{4}$ Department of Psychiatry, Athens \\ Medical School, Eginition Hospital; \\ ${ }^{5}$ Department of Neurology III, Aristotle \\ University of Thessaloniki, Greece
}

\section{Abstract}

Predicting the evolution of epilepsy is of obvious importance for patients and their families. Value of electroencephalography (EEG) is extensively used in the diagnosis of epilepsy yet its role as a prognostication method remains unclear. The aim of the present retrospective study is to investigate the relationship between serial EEG recordings and long-term clinical and social outcomes in a cohort of patients with epilepsy. Thirty-nine epileptic patients were monitored clinically and with repeat EEG recordings for more than 15 years. All patients who initially had epileptiform discharges ended up with poor or moderate seizure control whereas more than half of the patients with normal initial recordings had good clinical outcomes and satisfactory social adjustment. Deterioration of the recordings over time was associated with unfavourable results in a significant proportion of patients (90\%), while stable or improved EEG findings predicted a favourable outcome. It is concluded that serial EEG recordings can be used in the prognostic evaluation of epilepsy.

\section{Introduction}

The value of electroencephalography (EEG) for the diagnosis and classification of epilepsy, after fifty years of continuous worldwide application, is well established. In contrast, the prognostic value of EEG in epilepsy remains uncertain, with a number of studies providing contradictory results. ${ }^{1-8}$

Studies on neonates, children with West syndrome, and cases with febrile seizures suggested that EEG patterns had no predictive usefulness. ${ }^{9-11}$ Similarly, Hopkins et al. ${ }^{2}$ and Pohlmann-Eden et al. ${ }^{7}$ did not find the EEG to be of value in predicting seizure recurrence among patients presenting with a first epileptic seizure in adulthood.

Other studies, however, provided opposite results. Hughes and Fino, in a study on 1478 EEGs from 300 patients suffering from focal epilepsy, concluded that the EEG, especially the first and second recordings, can predict which patients will remain poorly controlled. ${ }^{12}$ In cases of seizure disorders that become evident at a later age, it has been reported that an epileptiform pattern in the initial recording has predictive value with regard to the future recurrence of seizures and the severity of the patient's clinical condition..$^{1,4}$ Finally, Donselaar et al. suggested that EEG findings may play a role in the decision to initiate or delay treatment after an idiopathic first seizure, as epileptic discharges in the first two EEGs were associated with a risk of recurrence of $83 \%$, vs. $12 \%$ in patients in whom both EEGs were normal. ${ }^{13}$

It is clear, therefore, that the important issue of the prognostic value of EEG in epilepsy remains unsettled. The present study contributes to this ongoing discussion by analyzing serial EEGs, performed during a 20 year period, and correlating EEG findings with longterm clinical and social outcomes in a cohort of patients with epilepsy.

\section{Materials and Methods}

Thirty-nine epileptic patients (22 males and 17 females, aged 41 to 57 at the end of the study) who were followed up at the EEG laboratory of the Department of Neurology, Athens Medical School, Eginition Hospital were included in the study. Patients were closely monitored clinically and electrophysiologically for at least 15 years and completed the monitoring period between the years 1999 and 2002 . All procedures were performed in compliance with institutional guidelines for the privacy of the subjects. The study has been approved by the ethical committee of the Aiginition Hospital.

All patients were diagnosed and evaluated for their epilepsy in our outpatient clinic. In accordance with our routine procedures, they had two regular appointments (in addition to extra visits due to emergency problems) and at least one EEG recording on a yearly basis. During this 15-year period, all patients showed particularly good compliance regularly attending their appointments, following closely medical instructions concerning their treatment and avoiding all seizure-provoking factors
Correspondence: Stylianos Gatzonis,

39 Sp.Merkouri str., Athens 11634, Greece. E-mail: sgatzon@med.uoa.gr

Key words: epilepsy, prognosis, electroencephalography, epilepsy outcome, seizure control, social adaptation.

Contributions: SG, NT and EA contributed to the research of patient files, EEG evaluation and final assessment of included patients, all of them participated in manuscript preparation; AS contributed to research of patient files and EEG evaluation; VK contributed to the final assessment of some patients, EEG evaluation and manuscript preparation; MK contributed to research of patient files, statistical analysis and manuscript preparation.

Conflict of interest: the authors report no conflicts of interest.

Received for publication: 21 March 2010.

Revision received: 12 October 2010.

Accepted for publication: 13 October 2010.

This work is licensed under a Creative Commons Attribution 3.0 License (by-nc 3.0).

@C Copyright S. Gatzonis et al., 2010

Licensee PAGEPress, Italy

Neurology International 2010; 2:e18

doi:10.4081/ni.2010.e18

such as sleep deprivation, alcohol consumption, seizure-provoking medications, prolonged exposure to intermittent light stimulation, and prolonged occupation with videogames or computers. Patients who did not fulfil the above criteria or patients with status epilepticus or any other neurological disorders of the central nervous system were excluded from the study.

In accordance with local regulations, all patients attending our clinic had their first epileptic seizure after the age of 15 years. Fourteen of our patients suffered from generalized seizures, and 25 from partial seizures with secondary generalisation. Fourteen patients had a history of febrile convulsions, head injuries at an earlier age or possible perinatal injury. None, however, had other neurological symptoms and signs or suffered from mental retardation, significant psychiatric or systemic disorders (i.e. diabetes mellitus, collagen disease, heart disease, etc.).

All patients' EEGs were recorded in the outpatient clinic, during morning sessions, with three EEG-recorders, a 12-channel Alvar Reega 2000, a 16-channel Era (Ote Biomedica), and an ElemaSchonander 16-channel Mingograf. Twenty-one electrodes were placed according to the 10-20 system. With the 16-channel machines, a monopolar and two dipolar montages (one longitudinal, one transverse) were recorded on paper. With the 12-channel machine, two longitudinal, 
two transverse, and one monopolar montage were used. Each session lasted $45 \mathrm{~min}$. No sleepdeprivation or sleep-induced recordings were included in the study.

\section{Classification of the electroence - phalography recordings}

In all cases, the first EEG was recorded before initiating drug treatment while subsequent EEGs were performed under antiepileptic drug therapy. We classified the initial EEG recordings, which were performed at the time of epilepsy onset, as follows: i) normal; ii) abnormal with specific epileptiform patterns such as spikes, spike-wave and polyspike-wave complexes, or sharp waves clearly distinguished from the background; six per second spike-waves, 14 and 6 per second positive spikes, and wicket spikes were considered as normal variants; or iii) abnormal with nonspecific findings (such as diffuse or focal slowing, attenuation or unilateral asymmetry of amplitude, or reactivity of rhythm).

\section{Evolution of the electroencepha - lography}

Deterioration of the recordings was considered to occur when: i) epileptiform patterns appeared in two or more of the subsequent recordings in those patients in whom no such patterns had been detected in the initial recordings, or ii) non-specific abnormalities appeared in more than half of the subsequent recordings in those patients in whom no such abnormalities had been detected in the initial recordings.

Improvement of the EEG recordings was considered to occur when: i) no epileptiform patterns appeared in the subsequent EEGs of those patients in whom such patterns had been detected in the initial recordings, or ii) non-specific findings appeared in 2 or fewer of the subsequent 14 recordings in those patients in whom such findings had been detected in the initial recordings.

\section{Clinical outcomes}

The clinical outcomes of the patients were assessed according to the frequency of their seizures. Thus, we classified patients' outcomes as satisfactory when seizure frequency was less than one seizure per 6 months, moderate when they had one or more seizures per 6 months but less than one seizure per month, and unsatisfactory when they consistently had more than one seizure per month.

\section{Social adjustment}

The social adjustment of the patients was assigned to one of three categories, based on an 18-degree scale. The evaluation was made according to the level of education they achieved (1-5), their form of employment (1-
3), whether they got married and had children or not (1-4), their financial situation (1-3), and their overall interpersonal relationships (1-3). Scores of 4-6 were characterized as poor social adjustment, scores of 7-12 as moderate, and scores of 13-18 as good.

\section{Statistical analysis}

Initial EEG findings are cross-classified to the evolution of the EEG patterns in Table 1, to the clinical outcome in Table 2 and social adjustment in Table 4. Clinical outcome is cross classified to the EEG evolution in Table 3, whereas type and relative severity of Epilepsy (deduced from the number of AEDs) is crossclassified to Gender in Table 5. Traditionally, in contingency tables analysis, the Pearson chisquare test is applied in order to test the independence of the cross-classifying characteristics. Since in our study the sample size is small, one cannot rely on the asymptotic Chisquare distribution of the test statistic under the hypothesis of independence and exact testing is needed. Therefore, throughout this paper, the Monte Carlo (MC) p-values, based on 10000 samples, instead of the asymptotic ones are calculated and used. The level of significance is set to 0.05 .

\section{Results}

Initial electroencephalography findings and electroencephalography evolution

The initial EEG, which was performed when seizures appeared for the first time, was: i) normal in 14 patients (35.8\%). In seven patients, the EEG became subsequently abnormal, disclosing specific epileptiform patterns in three patients and non-specific abnormal findings in the other four, while in the remain-

Table 1. Electroencephalography evolution.

\begin{tabular}{lcccc} 
Initial EEG & \multicolumn{2}{c}{ Evolution of EEG patterns } & \\
& Unchanged & Improved & Deteriorated & Total \\
Normal & $7(50 \%)$ & 0 & $7(50 \%)$ & 14 \\
Non-specific & $7(46.6 \%)$ & $4(26.6 \%)$ & $4(26.6 \%)$ & 15 \\
\hline Epileptiform & $6(60 \%)$ & $4(40 \%)$ & 0 & 10 \\
Total & 20 & 8 & 11 & 39 \\
\hline
\end{tabular}

Table 2. Clinical outcomes in relation to the initial electroencephalography.

\begin{tabular}{|c|c|c|c|c|}
\hline Initial EEG & $\begin{array}{l}\quad \text { Clinical 0 } \\
\text { Satisfactory } \\
\text { (seizures } \\
<1 / 6 \text { months) }\end{array}$ & $\begin{array}{l}\text { (according to seiz } \\
\text { Moderate } \\
\text { (seizures: } 1 / 6 \\
\text { months to } 1 / \mathrm{mo} \text { ) }\end{array}$ & $\begin{array}{l}\text { frequency) } \\
\text { Unsatisfactory } \\
\text { (seivures> } \\
\text { 1/ month) }\end{array}$ & Total \\
\hline Normal & $8(57 \%)$ & $4(29 \%)$ & $2(14 \%)$ & 14 \\
\hline Non-specific abnormal & $8(53.3 \%)$ & $3(20 \%)$ & $4(26.6 \%)$ & 15 \\
\hline Abnormal-epileptiform & 0 & $4(40 \%)$ & $6(60 \%)$ & 10 \\
\hline Total & 16 & 11 & 12 & 39 \\
\hline
\end{tabular}

Table 3. Clinical outcomes in relation to the electroencephalography evolution.

\begin{tabular}{lcccc} 
Clinical outcome & Unchanged & $\begin{array}{c}\text { EEG evolution } \\
\text { Improved }\end{array}$ & Deteriorated & Total \\
Satisfactory & $11(69 \%)$ & $4(25 \%)$ & $1(6 \%)$ & 16 \\
Moderate & $5(46 \%)$ & $2(18 \%)$ & $4(36 \%)$ & 11 \\
\hline Unsatisfactory & $4(33 \%)$ & $2(17 \%)$ & $6(50 \%)$ & 12 \\
Total & 20 & 8 & 11 & 39 \\
\hline
\end{tabular}

Table 4. Social adjustment of patients in relation to the initial electroencephalography.

\begin{tabular}{lcccc}
\hline Initial EEG & Good & $\begin{array}{c}\text { Social adjustment } \\
\text { Moderate }\end{array}$ & Poor & Total \\
Normal & $10(71 \%)$ & $4(29 \%)$ & 0 & 14 \\
Abnormal non-specific & $3(20 \%)$ & $11(73 \%)$ & $1(7 \%)$ & 15 \\
\hline Abnormal- epileptiform & 0 & $7(70 \%)$ & $3(30 \%)$ & 10 \\
Total & 13 & 22 & 4 & 39 \\
\hline
\end{tabular}


ing seven patients the EEG recordings remained constantly normal; ii) abnormal with non-specific findings in 15 patients (38.4\%). In four patients, specific epileptiform patterns appeared in the course of the serial EEGs that followed. In another four the initially non-specific abnormal findings gradually disappeared in the course of the 15 years, and in the remaining seven patients the EEGs remained unchanged; iii) abnormal with specific epileptiform patterns in 10 patients (25.6\%). In four of them the EEGs improved (i.e., the epileptiform patterns were no longer detectable but were replaced by non-specific findings), while in the other six patients the EEGs, over the 15 years of monitoring, consistently revealed specific epileptiform patterns (Table 1).

The cross-classification of the initial EEG findings vs the EEG evolution is provided in Table 1 and the above described relation between them is significant, since the hypothesis of independence is rejected for Table 1 (MC $\mathrm{P}=0.032$ ).

\section{Clinical outcome and electroence- phalography findings}

The clinical outcomes of the patients in relation to the initial EEGs are presented in Table 2. The group of patients that presented with normal and non-specific abnormal EEGs at the initial stage had statistically significantly better clinical outcome (satisfactory seizure control) compared to patients who presentedwith abnormal, specific epileptiform EEG patterns ( $\mathrm{MC} \mathrm{P}=0.032$ ).

Regarding the changes in the recordings over time, 20 of the EEGs remained the same, 11 deteriorated, and 8 improved (Table 3). Deterioration of the recordings was associated with an unfavourable clinical outcome (1 patient with satisfactory seizure control out of 11 with deteriorated recordings) while improved or unchanged recordings were associated with much better control of seizures (15 well-controlled patients out of 28 with stable or improved EEGs, MC $\mathrm{P}<0.05)$. Therefore, the development of novel abnormalities, particularly specific epileptiform discharges, in the follow-up recordings of patients with epilepsy has prognostic implications for their eventual outcome.

\section{Social adjustment and initial electroencephalography findings}

The educational and social profile of our patients at the end of the study was as follows: 18 had elementary education, 15 completed high school, and 6 received higher-level education; 5 patients were unemployed, 16 were occasionally employed, and 18 had permanent employment; 17 patients were married and 22 were single.

The initial EEG of the patients is not inde- pendent from their long-term social adjustment, since the hypothesis of independence is rejected in Table 4 ( $\mathrm{MC} \mathrm{P}<0.0005)$.

The group of patients with abnormal nonspecific and those with specific epileptiform EEG patterns do not differentiate in terms of their social adjustment (MC $\mathrm{P}=0.152$ ). Thus, patients who presented with normal EEG at the initial stage had statistically significantly better social adjustment (good) compared to patients who presented with non-specific abnormal and specific epileptiform EEG patterns ( $\mathrm{MC} \mathrm{P}<0.0005)$.

Finally, based on the results summarized in Table 5, we conclude that type and relative severity of epilepsy is unrelated to gender $(\mathrm{MCP}=1)$.

\section{Discussion}

The prediction of the long-term evolution of epilepsy is an issue of great significance for patients, care-givers and treating physicians alike. The early identification of patients destined to develop intractable epilepsy, in particular, has practical importance as it may critically influence decision-making regarding their treatment by allowing, for instance, timely surgical interventions or the application of innovative treatment strategies. Accordingly, there is a continuing interest for the development of reliable prognostic factors in epilepsy and over the years a number of clinical and laboratory variables of prognostic value have emerged, including EEG.

The present study contributes to this ongoing discussion by investigating the relationship between serial EEG recordings and longterm clinical outcome in 39 epileptic patients who were followed up for a period exceeding 15 years. In addition, we attempted to correlate initial EEG findings to long-term social adjustment, an issue that has not been previously addressed in relevant studies..$^{11-14}$

It is concluded that both initial and repeat EEG recordings carried useful information with regard to the long-term prognosis of these patients. In particular, all patients with epileptiform discharges $(n=10)$ in their initial EEGs ended up with poor or moderate seizure control and social outcomes. In contrast, more than half of the patients with normal initial recordings $(\mathrm{n}=14)$ had good clinical outcomes $(8 / 14,57 \%)$ and satisfactory social adjustment $(10 / 14,71 \%)$. Deterioration of the recordings over time was associated with unfavourable results in the majority of patients $(10 / 11,90 \%)$ while stable or improved EEG findings predicted a favourable outcome $(15 / 28,54 \%)$. In addition, the overall social adjustment of the patients, though obviously primarily dependent on factors such as seizure control, was also
Table 5. Type and relative severity of epilepsy (deduced from the number of AEDs) in relation to gender.

$\begin{array}{cccc} & \text { Males } & \text { Females Total } \\ \text { Generalized epilepsy/ } & 6 & 5 & 11\end{array}$

One AED

\begin{tabular}{lccc}
$\begin{array}{l}\text { Generalized epilepsy/ } \\
\text { Two or more AEDs }\end{array}$ & 2 & 1 & 3 \\
\hline $\begin{array}{l}\text { Partial epilepsy } \\
\text { One AED }\end{array}$ & 6 & 4 & 10 \\
$\begin{array}{l}\text { Partial epilepsy } \\
\text { Two or more AEDS }\end{array}$ & 8 & 7 & 15 \\
\hline Total & 22 & 17 & 39 \\
\hline
\end{tabular}

AED, antiepileptic drug.

influenced by the presence of even non-specific abnormalities in the initial recordings. Only $3 / 15$ (20\%) of these patients eventually had good adjustment, whereas in the majority $(11 / 15,73 \%)$ adjustment was moderate (MC $\mathrm{P}<0.05$ ).

There are certain methodological limitations in our study, including the relatively small number of investigated patients and its' retrospective nature, which necessitates the confirmation of our conclusions in a prospective manner. Despite these limitations, our data indicate that serial EEG recordings can be of use in the prognostic evaluation of epilepsy.

Previous studies on prognostic factors in epilepsy have provided limited information regarding EEG as most of them focused on clinical parameters. For instance, in three relevant large-scale studies, ${ }^{15-17}$ EEG was not included in the analysis. In addition, those studies that considered EEG as a prognostic factor provided controversial results. Elwes $e t$ $a l .{ }^{18}$ and the Collaborative Group for the Study of Epilepsy, ${ }^{19}$ concluded that the presence of epileptic, focal, slow-wave or background abnormalities in the pre-treatment EEG of patients with newly diagnosed epilepsy was devoid of prognostic value.

More recent studies, however, have emphasized the usefulness of EEG as a prognostic factor in epilepsy. Schreiner and PohlmannEden $^{20}$ suggested that the presence of focal epileptiform activity in the initial EEG after a first unprovoked seizure was a significant predictor of seizure recurrence. Shafer et $a l^{21}$ in a study of 306 patients with epilepsy concluded that the absence of generalized epileptiform activity was associated with epilepsy remission, defined as 5-year seizure freedom. Interestingly, in that study focal epileptiform activity in the initial EEG was associated with a good prognosis, perhaps due to the inclusion of a large number of patients with benign Rolandic epilepsy. Aikia et $a l^{3}{ }^{3}$ investigated, in a prospective study of 89 patients with newly diagnosed epilepsy, neurological, neuropsychological and EEG variables as potential predictors of seizure refractoriness. The authors 
concluded that a model incorporating 6 variables predicted correct seizure outcome in $94 \%$ of patients and emphasized that the major predictor of refractory seizures was the presence of a spike focus in the initial EEG. Finally, Ko et $a l .{ }^{4}$ retrospectively analyzed initial EEG findings and medical records of 184 epileptic children and concluded that diffuse slowing and focal spike and wave activity were independent predictors of seizure intractability.

Our results are certainly in line with these latter studies and indicate that patients with abnormal recordings at the time of diagnosis or with deteriorating recordings in the followup period end up with intractable seizures and social maladjustment. Therefore, the EEG, has early predictive value that may be helpful in identifying seizure intractability and developing appropriate treatment strategies.

\section{References}

1. Hughes JR, Kaydanova Y. Long-term studies on patients with absence and bilateral spike-wave complexes: 430 patients, up to 52 years follow-up. Clin Electroencephalogr 1997;28:193-206.

2. Hopkins A, Garman A, Clarke C. The first seizure in adult life. Value of clinical features, electroencephalography, and computerized tomography scanning in prediction of seizure recurrence. Lancet 1988;1:721-6.

3. Aikia M, Kalviainen R, Mervaala E, Riekinnen PJ Sr. Predictors of seizure outcome in newly diagnosed partial epilepsy: memory performance as a prognostic factor. Epilepsy Res 1999; 37:159-67.

4. Ko TS, Holmes GL. EEG and clinical predictors of medically intractable childhood epilepsy. Clin Neurophysiol 1999;110:124551.

5. Shinnar S, Berg AT, Moshe SL, et al. Discontinuing antiepileptic drugs in children with epilepsy: a prospective study. Ann Neurol 1994;35:534-45.

6. Lossius MI, Stavem K, Cjierstad L. Predictors for recurrence of epileptic seizures in a general epilepsy population. Seizure 1999;8:476-9.

7. Pohlmann-Eden B, Schreiner A, Mika A. Diagnostic and prognostic implications of the first epileptic seizure in adulthood. Fortschr Neurol Psychiatr 1994;62:147-54.

8. Hedstrom A, Olsson I. Epidemiology of absence epilepsy: EEG findings and their predictive value. Pediatr Neurol 1991;7: 100-4.

9. Ortibus EL, Sum JM, Hahn JS. Predictive value of EEG for outcome of epilepsy following neonatal seizures. Electroencephalogr Clin Neurophysiol 1996;98:175-85.

10. Kuturec M, Emoto SE, Sofijanov N, et al. Febrile seizures: is the EEG a useful predictor of recurrence? Clin Pediatr (Phila) 1997;36:31-6.

11. Haga Y, Watanabe K, Negoro T, et al. Do ictal, clinical and electroencephalographic features predict outcome in West syndrome? Pediatr Neurol 1995;13:226-9.

12. Hughes JR, Fino JJ. Focal seizures and EEG: prognostic considerations. Clin Electroencephalogr 2003;34:174-81.

13. van Donselaar CA, Schimsheimer RJ,
Geerts AT, Declerck AC. Value of the electroencephalogram in adult patients with untreated idiopathic first seizures. Arch Neurol1992;49:231-7.

14. Holmes MD, Dodrill CB, Wilkus RJ et al. Is partial epilepsy progressive? Ten year follow up of EEG and neuropsychological changes in adults with partial seizures. Epilepsia 1998;39:1189-93.

15. Berg AT, Levy SR, Novotny EJ, Shinnar S. Predictors of intractable epilepsy in childhood: a case-control study. Epilepsia 1996;37:24-30.

16. Camfield CS, Camfield P, Gordon K, et al. Outcome of childhood epilepsy: a population-based study with a simple scoring system for those treated with medication. J Pediatr 1993;122:861-8.

17. Sillanpaa M. Remission of seizures and prediction of intractability in long-term follow-up. Epilepsia 1993;34:930-6.

18. Elwes RDC, Johnson AL, Shorvon SD, Reynolds EH. The prognosis for seizure control in newly diagnosed epilepsy. $\mathrm{N}$ Engl J Med 1984;311:944-7.

19. Collaborative Group for the Study of Epilepsy. Prognosis of epilepsy in newly referred patients: a multicenter prospective study of the effects of monotherapy on the long-term course of epilepsy. Epilepsia 1992;33:45-51.

20. Schreiner P, Pohlmann-Eden B. Risk factors of seizure occurrence after a first unprovoked seizure. Epilepsia 1997;38:16.

21. Shafer SQ, Hauser WA, Annegers JF, Klass DW. EEG and other early predictors of epilepsy remission: a community study. Epilepsia 1988;29:590-600. 